\title{
Risques des rayonnements ionisants et normes de radioprotection
}

\author{
Introduction et conclusions du rapport de \\ I'ACADÉMIE DES SCIENCES*
}

(Rapport $n^{\circ}$ 23). Paris : Académie des sciences, 1989

\section{INTRODUCTION}

Les affections que peuvent causer les rayonnements ionisants sont bien connues depuis les observations effectuées sur les physiciens et les médecins qui ont été les pionniers de la radiologie. Nul ne peut, par exemple, oublier que Marie CURIE et sa fille Irène sont toutes deux mortes de leucémies provoquées par les rayons $X$ et les radioéléments qu'elles manipulaient sans grande précaution.

Dès 1928, la Commission internationale de protection contre les rayonnements ionisants a proposé des normes de radioprotection qui apparaîtraient aujourd'hui comme insuffisantes, mais qui néanmoins ont eu d'immenses mérites, par exemple celui de ramener à la normale, chez les médecins radiologistes, la fréquence des leucémies, alors qu'entre 1920 et 1940, elle était dix fois plus élevée que chez les médecins ne s'exposant pas aux rayonnements ionisants. Les normes actuellement en vigueur ont été preparées à la fin des années 1950. Leur but était de limiter le risque d'effets génétiques et de cancers radioinduits à un niveau considéré comme tolérable, en prenant comme référence les risques professionnels observés dans d'autres professions.

Depuis lors, les normes adoptées ont été en partie révisées en fonction d'acquisitions nouvelles. Les valeurs aujourd'hui admises ont été définies en 1977. En 1988, l'UNSCEAR, l'organe compétent des Nations Unies, a proposé de nouveaux facteurs de risque sur la base de nouvelles données concernant les survivants d'Hiroshima et Nagasaki. De ce fait, plusieurs groupes ont proposé de nouvelles normes qui ont suscité de vives discussions au plan international. En effet, l'adoption de normes trop basses handicaperait des activités médicales ou professionnelles.

\footnotetext{
* Composition de la Commission de l'Académie des sciences ayant établi ce rapport : J. BERNARD, président, A. BLANC-LAPIERRE, R. CASTAING, P. GALLE, R. LATARJET, M. TUBIANA. Le rapport est disponible auprès de l'Académie des sciences, 23, quai de Conti, 75006 Paris. Tél. : (1) 43266621 .
} 
Devant cette situation, M. Hubert CURIEN, ministre de la Recherche et de la Technologie a posé les deux questions suivantes au président de l'Académie des sciences :

1. L'évolution des connaissances justifie-t-elle une révision des normes de radioprotection ?

2. Que peut-on faire pour accroitre nos connaissances en ce domaine et, éventuellement améliorer la radioprotection de la population et des travailleurs?

L'Académie des sciences a alors constitué en son sein un groupe d'étude avec mission de faire la synthèse des données disponibles, en vue de répondre à ces deux questions. Le présent rapport, fruit des travaux de ce groupe, aborde les points suivants :

1. Les risques cancérogènes : les méthodes permettant d'aboutir à une estimation sont passées en revue ; on souligne les incertitudes qui grèvent l'évaluation des risques pour la vie entière. Ces risques sont d'abord estimés pour des irradiations uniques à doses moyennes* ( 1 à 2 sievert [Sv]).

2. Les effets mutagènes et tératogènes.

3. L'irradiation naturelle dont les variations peuvent être considérables. Les résultats de plusieurs enquêtes seront examinés.

4. L'estimation des risques à faibles doses (moins de $0,2 \mathrm{~Sv}$ ), délivrées à débit élevé ou faible, sachant que pour cette gamme de doses on ne dispose pratiquement pas de données humaines fiables.

5. La comparaison avec les risques de produits chimiques génotoxiques, à l'aide de quelques exemples.

6. Des recommandations concernant les études à entreprendre.

Le groupe qui a préparé ce rapport s'est réuni à plusieurs reprises en mai et en juin 1989. Les rédacteurs ont, de plus, procédé à l'audition d'experts et soumis le projet de rapport à plusieurs spécialistes français et étrangers. Ils ont tenu compte de leurs avis et les remercient ici de leur aide et de leurs critiques.

\section{CONCLUSIONS}

\section{RÉPONSES AUX QUESTIONS DE M. LE MINISTRE}

De tous les agents potentiellement toxiques de notre environnement, les rayonnements ionisants sont sans doute ceux dont les effets sont les mieux connus, et qui ont fait l'objet du plus grand nombre de travaux expérimentaux et d'enquêtes épidémiologiques. Tout naturellement,

* Classification des doses selon I'UNSCEAR : IUUNSCEAR a défini les faibles doses entre 0 et $0,2 \mathrm{~Sv}$. Les doses moyennes de 0,2 à $2 \mathrm{~Sv}$, les fortes doses de 2 à $10 \mathrm{~Sv}$ et les très fortes doses à plus de $10 \mathrm{~Sv}$. 
l'estimation quantitative du risque évolue avec l'accroissement des connaissances. D'où la récente proposition d'une révision des facteurs de risque et des normes, et les deux questions posées par le ministre à l'Académie des sciences au sujet d'une telle révision. Voici nos réponses :

\section{Première question : L'évolution des connaissances justifie-t-elle une révision des normes de radioprotection?}

Nous devons d'abord récapituler les facteurs d'imprécision dans l'évaluation du risque cancérogène des faibles doses délivrées à faibles débits. Nous les avons plusieurs fois soulignés, en particulier pages 43-45 de ce rapport. Les nouvelles évaluations du facteur de risque cancérogène reposent uniquement sur la surveillance des survivants des explosions atomiques d'Hiroshima et Nagasaki, lesquelles ont délivré des doses considérables aux plus forts débits réalisables. Les principales incertitudes auxquelles on se heurte dans l'évaluation du risque sont de deux types : celles liées à l'estimation du risque à forte dose ( $\geqq 2 S v$ ) et celles introduites par l'extrapolation du risque à forte dose vers le risque à faible dose.

a) Incertitudes spécifiques sur la dosimétrie des personnes exposées au cours des explosions d'Hiroshima et Nagasaki, dans la composition du rayonnement d'abord et, surtout de chaque individu compris dans les statistiques.

b) Les résultats d'enquêtes épidémiologiques sur les malades soumis à une irradiation dans un but thérapeutique ou diagnostique n'ont pas été pris en compte et la seule source de données considérée par I'UNSCEAR pour l'estimation des risques est celle des survivants d'Hiroshima et Nagasaki. Cette attitude paraît criticable. En effet, les malades pourralent fournir des informations importantes qui ne devraient pas être négligées. II n'y a en effet aucune raison de penser que le risque cancérogène chez les malades puisse être plus faible que chez les sujets sains.

c) Les analyses effectuées montrent que la méthodologie utilisée pour calculer le risque, notamment en ce qui concerne les modèles de projection et la normalisation des populations, pourrait entraîner des variations considérables dans l'estimation du facteur de risque. Un effort méthodologique serait indispensable.

d) Incertitudes sur le facteur de réduction quand on passe des forts aux faibles débits : les données actuelles ne nous permettent pas d'évaluer ce facteur de réduction avec une précision suffisante. D'une manière générale, ce facteur augmente lorsque le faible débit considéré s'écarte plus du fort débit de référence. II augmente aussi lorsque l'on fractionne l'irradiation. Nous savons aujourd'hui qu'il varie selon l'âge et le sexe du sujet considéré. Un facteur unique de réduction est donc nécessairement approximatif.

e) Incertitudes sur l'extrapolation des fortes doses vers les faibles doses : en radioprotection nous importent les effets des doses faibles, de l'ordre de 10 à $50 \mathrm{mSv}$ délivrées en des temps de l'ordre de l'année, le 
plus souvent en de multiples fractions. L'extrapolation effectuée à partir de doses au moins cent fois plus élévées (qui sont celles pour lesquelles on peut calculer le risque de facon fiable chez les survivants d'Hiroshima et Nagasaki), couvre les conditions d'incertitudes maximales. Nous somme assurés aujourd'hui que la relation dose-effet cancérogène n'est presque jamais linéaire, et qu'elle n'est pas la même pour tous les cancers. Dans ce cas, comme pour le débit de dose, un facteur unique d'extrapolation ne peut donc être qu'approximatif.

f) II apparaît que la notion de seuil pratique, jusqu'ici exclue par dogmatisme, ne saurait être rejetée a priori. Il semble qu'au-dessous de faibles valeurs de la dose, l'effet puisse être pratiquement nul, ou du moins négligeable en regard de la longévité moyenne de lhomme et du "bruit de fond" des cancers "naturels". On commence même à envisager que les radiations à très faibles doses pourraient avoir d'autres effets que nocifs.

g) Enfin les effets des radiations se combinent à ceux d'autres génotoxiques parfois plus importants (tabac, alcool, amiante, air pollué....), combinaisons qui conduisent à des situations très diverses.

Ces considérations nous amènent à apporter à la question posée les réponses suivantes :

1. II est possible que l'effet cancérogène d'une irradiation à faible dose, faible débit ou délivrée en plusieurs fractions ne soit pas supérieur, et puisse même être inférieur, à celui qui avait été estimé au moment où les normes de radioprotection actuellement en vigueur ont été adoptées.

2. Plusieurs enquêtes, actuellement en cours, pourraient, dans un délai de quelques années, fournir des données plus précises sur l'effet des faibles doses ou tout au moins donner une limite supérieure du risque. De ce point de vue, il faut insister sur l'intérêt des études analysant les conséquences des variations de l'irradiation naturelle, notamment en ce qui concerne le radon, dont les concentrations varient notablement. II nous paraîtrait donc prématuré de procéder à une révision des doses.

3. Les normes actuelles donnent déjà un niveau de sécurité appréciable. Des composés chimiques génotoxiques de grande diffusion ne font pas l'objet d'une rigueur comparable à celle qui a présidé à l'établissement de ces normes. L'effort à effectuer devrait être dirigé prioritairement vers ces composés chimiques.

4. Si, ultérieurement, des données témoignant de l'effet éventuel des faibles doses étaient apportées par les enquêtes en cours, les mesures corrélatives devraient avoir pour objet la réduction des doses au cours des examens de radiodiagnostic et surtout celle de la dose due au radon à l'intérieur des habitations, puisque ce sont là les deux sources majeures d'irradiation de la population française.

5. II apparaît justifié de limiter, autant qu'il est raisonnablement possible, l'exposition des travailleurs comme le prescrit la réglementation 
française de radioprotection édictée dans le cadre du Code du travail. L'introduction d'une dose limite pendant la carrière professionnelle mériterait d'être envisagée.

Seconde question : Que peut-on faire pour accroître nos connaissances en ce domaine et essentiellement améliorer la radioprotection de la population des travailleurs ?

1. En premier lieu, nous recommandons les études épidémiologiques sur les malades irradiés, études dont nous avons souligné les avantages (p. 49 et 50) : définition précise des conditions d'irradiation; dosimétrie exacte ; suivi médical minutieux pendant plusieurs décennies ; possibilité de réunir des nombres considérables de cas, et de poursuivre dans l'avenir aussi longtemps que l'on jugera bon ; facilité de réunir de bons témoins en grand nombre ; débits de dose moins éloignés que ceux d'Hiroshima-Nagasaki des faibles débits qui nous intéressent ici.

2. D'autres études épidémiologiques sont souhaitables, plusieurs étant d'ailleurs en cours :

- parmi les travailleurs exposés (p. 50), la vaste étude en cours au Centre international de recherche sur le cancer, qui porte sur plusieurs centaines de milliers de cas, donnera des résultats dans moins de deux ans. On devrait considérer la question en fonction de ces résultats ;

- il serait utile d'attendre également les premiers résultats de l'enquête soviétique en cours sur la population proche de Tchernobyl et sur celle du sud de l'Oural exposée à un accident nucléaire en 1957, qui devraient être disponibles dans un délai relativement court.

3. Nous recommandons de développer les études épidémiologiques se rapportant à l'irradiation naturelle, tout particulièrement au radon dans les habitations.

4. Nous recommandons toutes recherches sur la comparaison quantitative des risques de divers composés chimiques génotoxiques, en particulier de ceux quil sont émis par les combustions.

5. En ce qui concerne la recherche cancérologique fondamentale, on doit reconnaître qu'elle bénéficie déjà de puissantes motivations consécutives aux remarquables progrès récents en biologie moléculaire, en virologie, en immunologie et en cytogénétique. On pourrait inciter les principaux organismes (CNRS, INSERM) à s'intéresser à la radiobiologie trop souvent négligée.

Au total considérant que les normes actuelles paraissent déjà prudentes, qu'il n'existe aucune raison scientifique de les remettre en cause, que l'on pourrait en quelques années compléter les données sur les effets des faibles doses, il apparaît qu'un effort prioritaire devrait être effectué pour développer les recherches épidémiologiques sur les effets des faibles doses et qu'il serait préférable d'attendre le résultat de ces études pour procéder à une révision des normes. 